PHYSICAL REVIEW D 89, 126008 (2014)

\title{
$1 / N$ correction in the D3-brane description of a circular Wilson loop at strong coupling
}

\author{
E. I. Buchbinder \\ School of Physics, M013, University of Western Australia, 35 Stirling Highway, \\ Crawley, Western Australia 6009, Australia \\ A. A. Tseytlin* \\ The Blackett Laboratory, Imperial College, London SW7 2AZ, United Kingdom
}

(Received 29 April 2014; published 25 June 2014)

\begin{abstract}
We compute the one-loop correction to the probe D3-brane action in $A d S_{5} \times S^{5}$ expanded around the classical Drukker-Fiol solution ending on a circle at the boundary. It is essentially the logarithm of the one-loop partition function of an Abelian $\mathcal{N}=4$ vector multiplet in $A d S_{2} \times S^{2}$ geometry. This one-loop correction should be describing the subleading $1 / N$ term in the expectation value of circular Wilson loop in the totally symmetric rank $k$ representation in $S U(N)$ supersymmetric Yang-Mills theory at strong coupling. In the limit $1 \ll k \ll N$ when the circular Wilson loop expectation values for the symmetric representation and for the product of $k$ fundamental representations are expected to match, we find that this one-loop D3-brane correction agrees with the gauge theory result for the $k$-fundamental case.
\end{abstract}

DOI: $10.1103 /$ PhysRevD.89.126008

PACS numbers: 11.25.Tq, 11.25.-W

\section{INTRODUCTION AND SUMMARY}

Bogomolny-Prasad-Sommerfield (BPS) Wilson loops (WL) in higher representations in $\mathcal{N}=4$ super YangMills theory admit a dual description in terms of D-branes [1-6]. In particular, in [2] it was shown that WL in the symmetric representation of $S U(n)$ can be described in terms of D3-branes, with the number $k$ of boxes in the Young tableau playing the role of an extra parameter, in addition to $N$ and the 't Hooft coupling $\lambda$. For large $N$ and $k \ll N$ the dual description is given in terms of $k$ coincident strings (same as for multiply wrapped WL) while for $k \sim N \gg 1$ with $k / N=$ fixed it is expected to be in terms of a probe D3-brane in $A d S_{5} \times S^{5}$ space. $^{1}$

The classical probe D3-brane solution representing large $k$ circular WL found in [1] applies in the limit of large $N$ and large $\lambda$ with

$$
\kappa \equiv \frac{k \sqrt{\lambda}}{4 N}=\text { fixed }
$$

The WL expectation can be written as

$$
\langle W\rangle=e^{-\Gamma}, \quad \Gamma=\Gamma_{0}+\Gamma_{1}+\cdots,
$$

where $\Gamma_{0}$ is the D3-brane probe action evaluated on the classical solution [1]

$\Gamma_{0}=N \mathcal{F}_{0}, \quad \mathcal{F}_{0}=-2\left(\kappa \sqrt{1+\kappa^{2}}+\operatorname{Arcsinh} \kappa\right)$,

\footnotetext{
*Also at Lebedev Institute, Moscow.

${ }^{1}$ For $k \sim N^{2} \gg 1$ the probe approximation is no longer valid as one cannot ignore back reaction [7] of D-branes on geometry $[8,9]$.
}

and $\Gamma_{1}$ stands for the first subleading $1 / N$ correction. For $k \ll N$ one gets $\Gamma_{0}=-k \sqrt{\lambda}$ which is the result for $k$-wrapped circular string WL $[10,11] . \Gamma_{0}$ in $(1.3)$ was found to match the corresponding gauge theory matrix model results $[1,3]$ : at this leading order the matrix model expression [12-14] for circular WL in the fundamental representation extended to $k$-fundamental (or multiply wound) WL case [1] is the same as the WL corresponding to the symmetric representation $[3,4]$.

As for the subleading correction $\Gamma_{1}$, it appears to be nontrivial to extract it from the general result for the rank $k$ symmetric representation WL given in [15] but it can be readily found for the $k$-fundamental WL case. Starting with the matrix model solution of [13] and replacing $\sqrt{\lambda} \rightarrow k \sqrt{\lambda}$ one gets $[1]^{2}$

$$
\left\langle W_{\square^{k}}\right\rangle=e^{-\Gamma_{\square^{k}}}=N^{-1} e^{2 N \kappa^{2}} L_{N-1}^{1}\left(-4 N \kappa^{2}\right) .
$$

Here $L_{n}^{m}$ is the Laguerre polynomial, so that $\Gamma_{\square^{k}}(N, \kappa)$ satisfies [1]

$$
\begin{aligned}
& \Gamma_{\square^{k}} \equiv N \mathcal{F}, \\
&\left(\partial_{\kappa} \mathcal{F}\right)^{2}-N^{-1}\left(\partial_{\kappa}^{2} \mathcal{F}+3 \kappa^{-1} \partial_{\kappa} \mathcal{F}\right)-16\left(1+\kappa^{2}\right)=0 .
\end{aligned}
$$

For large $N$ and fixed $\kappa$ one finds

\footnotetext{
${ }^{2}$ Given the matrix model expression $\left\langle N^{-1} \operatorname{tr} e^{M}\right\rangle=$ $Z^{-1} \int[d M] N^{-1} \operatorname{tr} e^{M} \exp \left(-2 N \lambda^{-1} \operatorname{tr} M^{2}\right)$ for the WL expectation value in the fundamental representation, to find the expectation value in the tensor product of $k$ fundamental representations $\left\langle N^{-1} \operatorname{tr} e^{k M}\right\rangle$ one is to replace $k M \rightarrow M$, i.e., $\lambda \rightarrow k^{2} \lambda$. The result then depends on $N, k, \sqrt{\lambda}$ only through $N$ and $k \sqrt{\lambda}$, or $N$ and $\kappa$.
} 


$$
\mathcal{F}=\mathcal{F}_{0}(\kappa)+N^{-1} \mathcal{F}_{1}(\kappa)+O\left(N^{-2}\right),
$$

where $\mathcal{F}_{0}$ is the same as in (1.3) and the next correction is [16]

$$
\left(\Gamma_{1}\right)_{\square^{k}}=\mathcal{F}_{1}=\frac{1}{2} \ln \left(\kappa^{3} \sqrt{1+\kappa^{2}}\right) .
$$

In contrast to $\left\langle W_{\square^{k}}\right\rangle$ which depends only on $N$ and $k \sqrt{\lambda}$, the rank $k$ symmetric representation expression $\left\langle W_{\text {sym }_{k}}\right\rangle$ is, in general, a nontrivial function of the three parameters $N, \sqrt{\lambda}$, and $k$ [15]. The analog of the above expansion (1.6) will be to take $N$ large first for fixed $\kappa$ and $\sqrt{\lambda}$ and then expand in large $\sqrt{\lambda}$ at each order in $1 / N$.

There is no a priori reason why the leading large $\sqrt{\lambda}$ term in the first $1 / N$ correction in $\left\langle W_{\text {sym }_{k}}\right\rangle$ should be the same as (1.7). However, it is easy to see that in the limit $1 \ll k \ll N$, that is in the limit of small $\kappa$, the logarithms of the dimensions of the two representations $d_{\square k}=N^{k}$ and $d_{\text {sym }_{k}}=\frac{(N+k-1) !}{k !(N-1) !}$ are the same to leading order. Hence, using (1.7) one may expect that

$$
\kappa \ll 1:\left(\Gamma_{1}\right)_{\square^{k}} \approx\left(\Gamma_{1}\right)_{\mathrm{sym}_{k}} \approx \frac{1}{2} \ln \kappa^{3} .
$$

Our aim below will be to see how this result can be reproduced in the dual D3-brane description [1] of the symmetric representation WL. ${ }^{3}$

One natural suggestion is that to go beyond the leading large $N$ result (1.3) one should include the contribution of one-loop fluctuations of D3-brane fields near the classical solution of [1] (ignoring $\alpha^{\prime}$ corrections to the D3-brane probe action and also contributions of loops of massive string modes as they should be suppressed at leading order in large $\sqrt{\lambda}$ ). Since the D3-brane probe tension in $A d S_{5} \times$ $S^{5}$ is effectively proportional to $N$, the 1-loop correction $\Gamma_{1}$ to the D3-brane effective action (1.2) will be a function of $\kappa$ only. The path integral for the D3-brane probe action is not well defined in general (the Dirac-Born-Infeld action is nonrenormalizable), but the semiclassical correction may still make sense in the expansion near a BPS solution as it corresponds to taking into account the contributions of massless open string modes only. While the resulting expression for $\Gamma_{1}$ will be UV divergent, indicating the need to account for a proper embedding of this calculation into string theory, one may still expect that the finite $\kappa$ dependence is correctly captured (to leading order in large

\footnotetext{
${ }^{3}$ One can expect that the same conclusion should also hold in the case of the totally antisymmetric representation. It indeed holds for the leading semiclassical result at large $\lambda$ obtained in [3,4]: $\left\langle W_{A_{k}}\right\rangle=\exp \left(2 N \sqrt{\lambda} \sin ^{3} \theta / 3 \pi\right)$, where $\theta-\frac{1}{2} \sin 2 \theta=\frac{\pi k}{N}$. Taking the limit $k \ll N$ we find that $\left\langle W_{A_{k}}\right\rangle \rightarrow \exp (k \sqrt{\lambda})$ which coincides with the small $\kappa$ limit of (1.2), (1.3).
}

$\sqrt{\lambda}$ ) by the massless mode contributions only. Indeed, the string theory cutoff should be independent of the background parameter $\kappa$, and the massive string mode contributions should be suppressed by extra powers of the inverse of string tension $\sqrt{\lambda}$.

The investigation of the semiclassical quantization of the probe D3-brane action near the solution [1] representing the circular WL was initiated in $[17,18]$ which we will build on. The action for the quadratic fluctuations is essentially that of an Abelian $\mathcal{N}=4$ vector multiplet in $\operatorname{AdS}_{2} \times S^{2}$ background with $\kappa$-dependent radii. Computing the corresponding one-loop correction to the classical value of D3-brane action we will find that

$$
\begin{aligned}
\Gamma_{1} & =\frac{1}{2} \ln \left(L^{2} \Lambda^{2}\right)+\Gamma_{1 \text { fin }}(\kappa)+C_{1}, \\
\Gamma_{1 \text { fin }}(\kappa) & =\frac{1}{2} \ln \frac{\kappa^{3}}{\sqrt{1+\kappa^{2}}},
\end{aligned}
$$

where $\Lambda$ is UV cutoff, $L$ is $A d S_{5} \times S^{5}$ radius, and $C_{1}$ is a numerical ( $\kappa$-independent) constant given by the sum of $\zeta^{\prime}(0)$ terms for the corresponding quadratic fluctuation operators in $A d S_{2} \times S^{2}$ geometry.

We conclude that the $\kappa$-dependent part of the oneloop D3-brane result (1.9) differs in general from the $k$-fundamental WL expression (1.7), but the two do coincide in the small $\kappa$ limit, in agreement with (1.8). We conjecture that $\Gamma_{1 \text { fin }}$ in (1.9) should match the symmetric representation expression $\left(\Gamma_{1}\right)_{\text {sym }_{k}}$ for any finite $\kappa$.

Below in Sec. II A we shall first review the classical D3-brane solution [1] representing circular WL in the symmetric representation at strong coupling and then in Sec. II B describe the quadratic fluctuation part of the D3brane probe action expanded near it following $[17,18]$. The $\kappa$-parameter dependent part $\Gamma_{1 \text { fin }}(\kappa)$ of the corresponding one-loop effective action (1.9) will be found in Sec. II C.

In Appendix A we shall compute the numerical part $C_{1}$ of the effective action (1.9) using heat kernel methods [19-22] in $A d S_{2} \times S^{2}$ background. In Appendix B we shall review the structure of the one-loop correction [23] to circular WL in $A d S_{5} \times S^{5}$ string theory (corresponding to the limit of $N=\infty$ with $\lambda \gg 1$ for fixed $k$ ) and then rederive the result of [24] for its finite part for $k=1$ by completing the computation originally presented in [23] based on $A d S_{2}$ heat kernel expressions.

\section{ONE-LOOP CORRECTION NEAR THE D3-BRANE SOLUTION}

\section{A. Classical solution}

Let us first review the classical solution [1] for D3-brane probe in $A d S_{5} \times S^{5}$ that should be describing [2] the expectation value of a BPS circular Wilson loop in the rank $k$ symmetric representation of $S U(N)$. The D3-brane is localized in $A d S_{5}$ with coordinates chosen as 


$$
\begin{aligned}
d s^{2}= & \frac{L^{2}}{\sin ^{2} \eta}\left[d \eta^{2}+\cos ^{2} \eta d \psi^{2}+d \rho^{2}\right. \\
& \left.+\sinh ^{2} \rho\left(d \theta^{2}+\sin ^{2} \theta d \phi^{2}\right)\right] .
\end{aligned}
$$

Here $L$ is the $A d S_{5} \times S^{5}$ radius, $L^{2}=\left(4 \pi g_{s} N\right)^{1 / 2} \alpha^{\prime} \equiv$ $\sqrt{\lambda} \alpha^{\prime}$. The world volume of the D3 brane will be parametrized by $\sigma^{\alpha}=(\psi, \rho, \theta, \phi)$. The bosonic part of the D3-brane probe action in $A d S_{5} \times S^{5}$ is

$$
\begin{aligned}
S & =T_{D_{3}}\left(\int d^{4} \sigma e^{-\Phi} \sqrt{\operatorname{det}\left(g+2 \pi \alpha^{\prime} F\right)}-\int C_{4}\right), \\
T_{D_{3}} & =\frac{1}{(2 \pi)^{3} g_{s} \alpha^{\prime 2}}=\frac{N}{2 \pi L^{4}} .
\end{aligned}
$$

The relevant solution ending on a circle parametrized by $\psi$ at the boundary $\eta=0$ is $[1]^{4}$

$$
\begin{aligned}
\sin \eta & =\kappa^{-1} \sinh \rho, \quad 2 \pi \alpha^{\prime} \bar{F}_{\psi \rho}=\frac{i L^{2}}{\sinh ^{2} \rho}, \\
\kappa & =\frac{k \sqrt{\lambda}}{4 N}=\frac{k L^{2}}{4 N} .
\end{aligned}
$$

The induced metric on the D3-brane is then that of $A d S_{2} \times S^{2}$

$$
\begin{aligned}
d \bar{s}^{2}= & a_{A d S_{2}}^{2}\left(d \xi^{2}+\sinh ^{2} \xi d \psi^{2}\right)+a_{S^{2}}^{2}\left(d \theta^{2}+\sin ^{2} \theta d \phi^{2}\right) \\
a_{A d S_{2}} & =L \sqrt{1+\kappa^{2}}, \quad a_{S^{2}}=L \kappa \\
\sinh \xi & =\left(1+\kappa^{2}\right)^{-1 / 2} \cot \eta
\end{aligned}
$$

Substituting the solution (2.3) into the action (2.2) (and taking into account boundary terms) one finds that the latter is equal to $\Gamma_{0}$ in (1.3) [1].

Let us note that the BPS solution corresponding to the ( $k$-fold) infinite straight line WL can be found from (2.3) by taking $\eta$ and $\rho$ small and replacing $\psi$ with a noncompact coordinate $x$. Both circle and straight line solutions can be described in a unified manner in the coordinate system where the $A d S_{5}$ metric is [17]

$$
d s^{2}=L^{2}\left(d u^{2}+\cosh ^{2} u d s_{A d S_{2}}^{2}+\sinh ^{2} u d s_{S^{2}}^{2}\right) .
$$

Choosing $d s_{A d S_{2}}^{2}$ in Poincaré coordinates, i.e., $d s_{\widetilde{A d S_{2}}}^{2}=r^{-2}\left(d \mathrm{x}^{2}+d r^{2}\right)$, corresponds to the straight line case and choosing it in global coordinates $d s_{A d S_{2}}^{2}=$ $d \chi^{2}+\sinh ^{2} \chi d \psi^{2}$ - to the circle case. Then in both cases

\footnotetext{
${ }^{4}$ The gauge field has an extra factor of $i$ in Euclidean space (it becomes a real electric field in Minkowski signature). The momentum conjugate to $A_{\psi}$ component of vector potential is set equal to $k$ which on the dual gauge theory side is identified with the rank of the symmetric representation.
}

the solution is simply $u=u_{\kappa}, \sinh u_{\kappa}=\kappa$, so that the induced metric [cf. (2.4)] and the electric gauge field background are

$$
\begin{gathered}
\bar{g}_{\alpha \beta} d \sigma^{\alpha} d \sigma^{b}=L^{2}\left(\cosh ^{2} u_{\kappa} d s_{A d S_{2}}^{2}+\sinh ^{2} u_{\kappa} d s_{S^{2}}^{2}\right), \\
\sinh u_{\kappa}=\kappa \\
2 \pi \alpha^{\prime} \bar{F}=i L^{2} \cosh u_{\kappa} e^{0} \wedge e^{1}
\end{gathered}
$$

where $\left(e^{0}, e^{1}\right)$ is the vielbein of the Euclidean $A d S_{2}$.

Let us recall that the Euclidean $A d S_{2}$ space in Poincaré coordinates with boundary $R$ (which we shall denote as $\widetilde{A d S}_{2}$ ) is not globally equivalent to $H_{2}=A d S_{2}$ in global coordinates with boundary $S^{1}$. In particular, their regularized and renormalized volumes are different

$V_{\widetilde{A d S_{2}}}=\frac{T a}{\epsilon} \rightarrow 0, \quad V_{A d S_{2}}=2 \pi a^{2}\left(\frac{1}{\varepsilon}-1\right) \rightarrow-2 \pi a^{2}$.

Here $a$ is the radius, $T$ is the length of the boundary of $\widetilde{A d S_{2}}$ and $\varepsilon$ is a radial IR cutoff.

In what follows we shall always assume, as by now standard, that all power IR divergences are ignored (being cancelled by proper boundary terms), so that volumes should be replaced by their renormalized values. As a result, in the straight line WL case the classical D3-brane probe action vanishes [1] (consistent with $\langle W\rangle=1$ in this $1 / 2$ BPS case), while in the circular WL case one finds a nontrivial expression (1.3). This is, of course, parallel to what happens in the corresponding string theory description of these WL's at finite $k$ [11,23-25]. The same will also apply at the loop level.

\section{B. Quadratic fluctuation action}

Our aim is to compute the first quantum correction to the D3-brane effective action expanded near the above classical solution. As the D3-brane action (2.2) scales as $N$ (for fixed $L$ ), the one-loop correction corresponds to the first subleading order in $1 / N$ expansion at fixed $\kappa$.

The derivation of the quadratic fluctuation action was described in detail in $[17,18]$ which we follow here. In a staticlike gauge the remaining 6 bosonic scalar fluctuations are $\Phi^{I}=L\left(\delta u, \delta \theta^{a}\right)$ where $u=u_{\kappa}+T_{D_{3}}^{-1 / 2} \delta u$ and $\delta \theta^{a}$ are 5 fluctuations in $S^{5}$ directions. There are also gauge field fluctuations defined by $2 \pi \alpha^{\prime} F_{\alpha \beta}=2 \pi \alpha^{\prime} \bar{F}_{\alpha \beta}+T_{D_{3}}^{-1 / 2} f_{\alpha \beta}$. The quadratic fluctuation action is the sum of the bosonic and fermionic parts $\tilde{S}=\tilde{S}_{B}+\tilde{S}_{F}$ and is essentially the same as the action of an Abelian $\mathcal{N}=4$ supersymmetric vector multiplet in curved $A d S_{2} \times S^{2}$ background $^{5}$ :

\footnotetext{
${ }^{5}$ The simplicity of the fluctuation action is due to the residual supersymmetry of the background BPS solution.
} 


$$
\begin{aligned}
\tilde{S}_{B} & =\int d^{4} \sigma \sqrt{\bar{M}}\left(\frac{1}{2} G^{\alpha \beta} \partial_{\alpha} \Phi^{I} \partial_{\beta} \Phi^{I}+\frac{1}{4} G^{\alpha \beta} G^{\gamma \delta} f_{\alpha \gamma} f_{\beta \delta}\right), \\
& =\mathrm{c} \int d^{4} \sigma \sqrt{G}\left(\frac{1}{2} G^{\alpha \beta} \partial_{\alpha} \Phi^{I} \partial_{\beta} \Phi^{I}+\frac{1}{4} G^{\alpha \beta} G^{\gamma \delta} f_{\alpha \gamma} f_{\beta \delta}\right),
\end{aligned}
$$

$$
\tilde{S}_{F}=\int d^{4} \sigma \sqrt{\bar{M}} G^{\alpha \beta} \Theta\left(i \Gamma_{\alpha} \nabla_{\beta}\right) \Theta=\mathrm{c} \int d^{4} \sigma \sqrt{G} \Theta\left(i \Gamma^{\alpha} \nabla_{\alpha}\right) \Theta .
$$

Here $\bar{M}$ is the determinant of the matrix

$$
\begin{gathered}
\bar{M}_{\alpha \beta}=\bar{g}_{\alpha \beta}+2 \pi \alpha^{\prime} \bar{F}_{\alpha \beta}, \\
\sqrt{\bar{M}}=\mathrm{c} \sqrt{G}, \quad \mathrm{c} \equiv \operatorname{coth} u_{k}=\frac{\sqrt{1+\kappa^{2}}}{\kappa},
\end{gathered}
$$

where $\bar{g}_{\alpha \beta}$ and $\bar{F}_{\alpha \beta}$ are the induced metric and the classical solution for the gauge field. $G$ is the determinant of the "open string" metric

$$
G_{\alpha \beta}=\bar{g}_{\alpha \beta}+\left(2 \pi \alpha^{\prime}\right)^{2} \bar{g}^{\gamma \delta} \bar{F}_{\alpha \gamma} \bar{F}_{\beta \delta},
$$

which is the inverse of the symmetric part of $\bar{M}_{\alpha \beta}$, i.e., $\bar{M}^{(\alpha \beta)}=G^{\alpha \beta}$ (see, e.g., [26]). In the present case $G_{\alpha \beta}$ turns out to be that of the $A d S_{2} \times S^{2}$ space with equal radii [cf. (2.7)]

$$
G_{\alpha \beta} d \sigma^{\alpha} d \sigma^{\beta}=a^{2}\left(d s_{A d S_{2}}^{2}+d s_{S^{2}}^{2}\right), \quad a=L \kappa .
$$

The spinor covariant derivative $\nabla_{\alpha}$ in (2.11) is defined with respect to $G_{\alpha \beta}$.

The action (2.10), (2.11) is different from the action for a massless $\mathcal{N}=4$ vector multiplet in $A d S_{2} \times S^{2}$ geometry only by the overall constant factor c. The presence of this factor which originates from $\sqrt{\bar{M}}$ in (2.13) implies that the covariant measures for the fluctuations in question (which are essentially massless open-string modes) should be defined with respect to $\bar{M}_{\alpha \beta}$ as ${ }^{6}$

$$
\begin{gathered}
\|\Phi\|^{2}=\int d^{4} \sigma \sqrt{\bar{M}} \Phi^{I} \Phi^{I}=\mathrm{c} \int d^{4} \sigma \sqrt{G} \Phi^{I} \Phi^{I} \\
\|a\|^{2}=\int d^{4} \sigma \sqrt{\bar{M}} M^{\alpha \beta} a_{\alpha} a_{\beta}=\mathrm{c} \int d^{4} \sigma \sqrt{G} G^{\alpha \beta} a_{\alpha} a_{\beta} \\
\|\Theta\|^{2}=\int d^{4} \sigma \sqrt{\bar{M}} \Theta \cdot \Theta=\mathrm{c} \int d^{4} \sigma \sqrt{G} \Theta \cdot \Theta
\end{gathered}
$$

\footnotetext{
${ }^{6}$ Here $a_{\alpha}$ is a vector potential, $f_{\alpha \beta}=\partial_{\alpha} a_{\beta}-\partial_{\beta} a_{\alpha}$.
}

\section{One-loop correction to D3-brane effective action}

In general, the one-loop effective action of the $\mathcal{N}=4$ vector multiplet theory in curved background is given by the standard sum of the gauge field, 6 real scalar and 4 Majorana fermion contributions

$$
\Gamma_{1}=\Gamma_{g}+6 \Gamma_{s}+4 \Gamma_{f}
$$

$\Gamma_{g}=\frac{1}{2} \ln \operatorname{det}\left(-G_{\alpha \beta} \nabla^{2}+R_{\alpha \beta}\right)-\ln \operatorname{det}\left(-\nabla^{2}\right)$,

$\Gamma_{s}=\frac{1}{2} \ln \operatorname{det}\left(-\nabla^{2}+\frac{1}{6} R\right)$,

$\Gamma_{f}=-\frac{1}{2} \ln \operatorname{det}\left(i \Gamma^{\alpha} \nabla_{\alpha}\right)=-\frac{1}{4} \ln \operatorname{det}\left(-\nabla^{2}+\frac{1}{4} R\right)$,

where the second term in $\Gamma_{g}$ corresponds to the contribution of the ghosts. In the present case of equal-radii conformally-flat $A d S_{2} \times S^{2}$ geometry (2.15) the Ricci scalar and the Weyl tensor of $G_{\alpha \beta}$ vanish. ${ }^{7}$ The above determinants are assumed to be defined with respect to the measures in (2.16)-(2.18).

Since $A d S_{2} \times S^{2}$ is a homogeneous space, $\Gamma_{1}$ will be proportional to its volume. As already mentioned above, we shall assume that all power IR divergences should be ignored, i.e., the volume of $A d S_{2} \times S^{2}$ space with boundary $S^{1} \times S^{2}$ should be replaced by its renormalized value

$$
V_{A d S_{2} \times S^{2}}=\left(4 \pi a^{2}\right) \times\left(-2 \pi a^{2}\right)=-8 \pi^{2} a^{4} .
$$

In the straight-line solution case the renormalized volume will vanish [see (2.9)], $V_{\widetilde{A d S_{2}} \times S^{2}}=0$, and thus, as in the corresponding string-theory description [23,24], the one- (and higher-) loop corrections will vanish too.

In the circular solution case $\Gamma_{1}$ will be nonzero and will contain three terms [cf. (1.9)]:

(i) a UV divergent (conformal anomaly related) term $\Gamma_{\infty}$ proportional to the logarithm of the product of (e.g., proper-time $\epsilon=\Lambda^{-2} \rightarrow 0$ ) UV cutoff and the radius $a$ in (2.15);

(ii) a finite contribution $\Gamma_{m} \sim \ln \mathrm{c}$ of the nontrivial measure;

(iii) a nontrivial numerical constant $C_{1}$ (given by the sum of finite parts of $\operatorname{logs}$ of normalized determinants) that can be found using standard heat kernel techniques [20] (as discussed for $4 \mathrm{~d}$ scalars, vectors, and spinors in $A d S_{2} \times S^{2}$ background in a different context in $[21,22])$.

We will present the computation of $C_{1}$ in Appendix A while here will concentrate on the first two contributions. Explicitly, we have

\footnotetext{
${ }^{7}$ Thus the scalars $\Phi^{I}$ in (2.10) are, in fact, conformally coupled.
} 
$\Gamma_{\infty}=-\frac{1}{2} B_{4} \ln \left(a^{2} \Lambda^{2}\right), \quad B_{4}=\frac{1}{(4 \pi)^{2}} \int d^{4} \sigma \sqrt{G} b_{4}$,

where $b_{4}$ is the local Seeley coefficient (see, e.g., [27,28]). In the present $A d S_{2} \times S^{2}$ case

$$
\begin{gathered}
b_{4}=-\mathrm{a} R^{*} R^{*}, \quad R^{*} R^{*}=-2 R_{\alpha \beta}^{2}=-8 a^{-4}, \\
\mathrm{a}=\mathrm{a}_{g}+6 \mathrm{a}_{s}+4 \mathrm{a}_{f}=\frac{31}{180}+6 \times \frac{1}{360}+4 \times \frac{11}{720}=\frac{1}{4} \\
B_{4}=\frac{1}{(4 \pi)^{2}} V_{A d S_{2} \times S^{2}} b_{4}=-1
\end{gathered}
$$

where we used (2.22). Thus (2.23) becomes

$$
\Gamma_{\infty}=\frac{1}{2} \ln \left(a^{2} \Lambda^{2}\right) .
$$

The regularized measure contribution is also controlled by the same Seeley coefficient $B_{4}$ appearing in the expansion of $\operatorname{tr} 1 \rightarrow \operatorname{tr} e^{-\epsilon \Delta}$ ( $\Delta$ is the corresponding 2 nd order operator $),{ }^{8}$ i.e.,

$$
\Gamma_{m}=\frac{1}{2} B_{4} \ln \mathrm{c}=-\frac{1}{2} \ln \mathrm{c} .
$$

Since the UV cutoff should not depend on the parameter $\kappa$ of the background solution (it should be, in fact, proportional to $\alpha^{\prime-1}$ in a consistent string theory embedding) we can then determine the $\kappa$-dependent part of $\Gamma_{1}$ by combining (2.27) and (2.28) and using the definitions of c (2.13) and $a$ (2.15):

$$
\begin{aligned}
\Gamma_{\infty}+\Gamma_{m} & =\frac{1}{2} \ln \left(L^{2} \Lambda^{2}\right)+\Gamma_{1 \text { fin }}(\kappa), \\
\Gamma_{1 \text { fin }}(\kappa) & =\frac{1}{2} \ln \frac{\kappa^{3}}{\sqrt{1+\kappa^{2}}} .
\end{aligned}
$$

We thus find the expression in (1.9) announced in the Introduction.

\section{ACKNOWLEDGMENTS}

We would like to thank N. Drukker, B. Fiol, M. Kruczenski, Yu. Makeenko, and A. Tirziu for very useful

\footnotetext{
${ }^{8}$ Note that the squared fermionic operator is assumed to be defined with respect to the same measure (2.18). Then all power divergences cancel, consistent with supersymmetry of this model. In general, given a spectral problem for an operator $\mathcal{O}$ with respect to the measure $\mu$, i.e., $\mathcal{O} f_{n}=\lambda_{n} f_{n}, \int \mu f_{n}^{*} f_{m}=\delta_{n m}$, the path integral with the action $S=\int \mu \phi^{*} \mathcal{O} \phi$ is expressed in terms of $\operatorname{det} \mathcal{O}=\prod_{n} \lambda_{n}$ (one sets $\phi=\sum_{n} c_{n} f_{n}$ and integrates over $c_{n}$ ). Then $\operatorname{det} \mathcal{O}^{2}=\prod_{n} \lambda_{n}^{2}$ corresponds to path integral with the action $S=\int \mu \phi^{*} \mathcal{O}^{2} \phi$ and the constant measure factor dependence is controlled by the Seeley coefficient of $\mathcal{O}^{2}$ (see, e.g., Appendix A in [23]).
}

discussions. The work of E. I. B. was supported by the ARC Future Fellowship FT120100466. The work of A. A. T. was supported by the ERC Advanced Grant No. 290456 and also by the STFC Grant ST/J000353/1. This work was also supported in part by the ARC Discovery Project No. DP140103925. E. I. B. would like to thank Theory Group at Imperial College where the part of the work was done for warm hospitality.

\section{APPENDIX A: FINITE PART OF ONE-LOOP EFFECTIVE ACTION OF $\mathcal{N}=4$ VECTOR MULTIPLET ON $A d S_{2} \times S^{2}$}

The contributions of determinants of 2 nd order operators in (2.20), (2.21) can be expressed in terms of the trace of the corresponding heat kernel in the standard way. In the present case of $A d S_{2} \times S^{2}$ space (2.15)

$\frac{1}{2} \ln \operatorname{det} \Delta=-\frac{1}{2} \int_{\epsilon}^{\infty} \frac{d t}{t} \mathrm{~K}(t), \quad \mathrm{K}(t)=V_{A d S_{2} \times S^{2}} K(t)$.

Here $\mathrm{K}(t)=\sum_{n} e^{-\lambda_{n} t}$ and for a homogeneous space $K(t) \equiv K(x, x ; t)=\sum_{n} e^{-\lambda_{n} t} f_{n}^{*}(x) f_{n}(x)$ does not depend on the point $x\left(\left\{f_{n}(x)\right\}\right.$ is a set of normalized eigenfunctions of $\Delta$ with eigenvalues $\lambda_{n}$ ). The renormalized expression for the $A d S_{2} \times S^{2}$ volume factor was given in (2.22).

In general, the finite part of (A1) is naturally expressed as $-\frac{1}{2} \zeta^{\prime}(0)$ in terms of the corresponding $\zeta$-function

$\zeta(z)=\frac{1}{\Gamma(z)} \int_{0}^{\infty} d t t^{z-1} \mathrm{~K}(t)=\frac{1}{\Gamma(z)} V_{A d S_{2} \times S^{2}} \int_{0}^{\infty} d t t^{z-1} K(t)$,

$$
\frac{1}{2} \ln \operatorname{det} \Delta=-\frac{1}{2} \zeta(0) \ln (1 / \bar{\epsilon})-\frac{1}{2} \zeta^{\prime}(0) .
$$

Here $\bar{\epsilon}=a^{-2} \mu \epsilon$ is the combination of the UV cutoff $\epsilon=\Lambda^{-2}$, geometrical scale $a$ and a possible measure factor $\mu$ (corresponding to $\Delta \rightarrow \mu \Delta$ ). If we formally include in $\zeta(0)$ the contribution of potential zero modes (by IRregularizing them with a small mass term) its expression will match the value of the Seeley coefficient $B_{4}$ in (2.23), (2.28).

For a product space like $A d S_{2} \times S^{2}$ the scalar and spinor heat kernel factorizes. For completeness, let us first quote the "untraced" expressions for heat kernels $K\left(x, x^{\prime} ; t\right)$ of massless scalar Laplacians on $S^{2}$ and $A d S_{2}$ (see, e.g., $[19,29,30])$. They depend on coordinates of two points $x, x^{\prime}$ only through the corresponding geodesic distance $d\left(x, x^{\prime}\right)$. For $S^{2}$ with unit-radius metric $d s^{2}=d \theta^{2}+\sin ^{2} \theta d \phi^{2}$, 
$x=(\theta, \phi)$, the geodesic distance is given by $\cos d\left(x, x^{\prime}\right)=$ $\cos \theta \cos \theta^{\prime}+\sin \theta \sin \theta^{\prime} \cos \left(\phi-\phi^{\prime}\right)$ and $^{9}$

$K_{S^{2}}\left(x, x^{\prime} ; t\right)=\frac{1}{4 \pi} \sum_{\ell=0}^{\infty}(2 \ell+1) P_{\ell}\left(\cos d\left(x, x^{\prime}\right)\right) e^{-t \ell(\ell+1)}$.

For $A d S_{2}$ with metric $d \xi^{2}+\sinh ^{2} \xi d \psi^{2}, x=(\xi, \psi)$, the geodesic distance is $\cosh d\left(x, x^{\prime}\right)=\cosh \xi \cosh \xi^{\prime}-$ $\sinh \xi \sinh \xi^{\prime} \cos \left(\psi-\psi^{\prime}\right)$ and ${ }^{10}$

$$
\begin{aligned}
K_{A d S_{2}}\left(x, x^{\prime} ; t\right)= & \frac{1}{2 \pi} \int_{0}^{\infty} d v v \tanh (\pi v) \\
& \times P_{-\frac{1}{2}+i v}\left(\cosh d\left(x, x^{\prime}\right)\right) e^{-t\left(v^{2}+\frac{1}{4}\right)} .
\end{aligned}
$$

Here $P_{q}(y)$ is the Legendre function that becomes equal to 1 at coincident points when $d(x, x)=0$, i.e., $P_{q}(1)=1$. Let us note that $K_{\text {ddS }_{2}}\left(x, x^{\prime} ; t\right)$ admits also an alternative representation [29]:

$$
\begin{aligned}
& K_{A d S_{2}}\left(x, x^{\prime} ; t\right) \\
& \quad=\frac{\sqrt{2}}{(4 \pi t)^{3 / 2}} \int_{d\left(x, x^{\prime}\right)}^{\infty} d u \frac{u}{\sqrt{\cosh u-\cosh d\left(x, x^{\prime}\right)}} e^{-\frac{1}{4}\left(\frac{u^{2}}{t}+t\right)} .
\end{aligned}
$$

The expressions for $K(t)=K(x, x ; t)$ for the massless scalar, vector, and fermion operators in (2.20), (2.21) can be found in [19-22] and are summarized below. For a real scalar operator on $A d S_{2} \times S^{2}$ with equal radii $a$ one has

$$
\begin{gathered}
K^{\mathrm{s}}(t)=K_{A d S_{2}}^{\mathrm{s}}(t) K_{S^{2}}^{\mathrm{s}}(t), \\
K_{S^{2}}^{\mathrm{s}}(t)=\frac{1}{4 \pi a^{2}} \sum_{\ell=0}^{\infty}(2 \ell+1) e^{-t \ell(\ell+1) a^{-2}}, \\
K_{A d S_{2}}^{\mathrm{s}}(t)=\frac{1}{2 \pi a^{2}} \int_{0}^{\infty} d v v \tanh (\pi v) e^{-t\left(v^{2}+\frac{1}{4}\right) a^{-2}} .
\end{gathered}
$$

\footnotetext{
${ }^{9}$ The derivation starts with $K$ written as a sum of product of normalized eigenfuctions (spherical harmonics) and uses the summation formula for the associate Legendre polynomials: $P_{\ell}\left(\cos d\left(x, x^{\prime}\right)\right)=P_{\ell}(\cos \theta) P_{\ell}\left(\cos \theta^{\prime}\right)+2 \sum_{m=1}^{\ell} \frac{(\ell-m) !}{(\ell+m) !} P_{\ell}^{m}(\cos \theta) \times$ $P_{\ell}^{m}\left(\cos \theta^{\prime}\right) \cos \left(\phi-\phi^{\prime}\right), \quad$ with $\quad P_{\ell}^{-m}(\cos \theta)=(-1)^{m} \frac{(\ell-m) !}{(\ell+m) !}$ $P_{\ell}^{m}(\cos \theta)$.

${ }^{10}$ The expressions (A4) and (A5) are formally related by an analytic continuation and use of the relation $\sum_{\ell} f(\ell)=$ $\frac{1}{2 i} \int_{C} d z \cot (\pi z) f(z)$ where $C$ encircles the real axis. Explicitly, one is to set $\ell \rightarrow z=-\frac{1}{2}+i v$, continue the angles $\theta \rightarrow i \xi, \phi \rightarrow$ $\psi$ so that $d_{S^{2}}\left(x, x^{\prime}\right) \rightarrow d_{A d S_{2}}\left(x, x^{\prime}\right)$, and finally reverse the overall sign of the metric (or restore the radius factor and set $a \rightarrow i a$ ). The latter corresponds to $t \rightarrow-t$ in (A4), as required to match (A5).
}

Here $v$ is a continuous spectral parameter for the Euclidean $A d S_{2}$ case. ${ }^{11}$ From now on we shall set the radius $a=1$ as the dependence on it is controlled by the conformal anomaly coefficient $B_{4}$ and was already determined in (2.27).

For a single $4 \mathrm{~d}$ Majorana fermion contribution one gets

$$
\begin{array}{r}
K^{\mathrm{f}}(t)=-\frac{1}{2} K_{A d S_{2}}^{\mathrm{f}}(t) K_{S^{2}}^{\mathrm{f}}(t), \\
K_{S^{2}}^{\mathrm{f}}(t)=-\frac{1}{2 \pi} \sum_{\ell=0}^{\infty}(2 \ell+2) e^{-t(\ell+1)^{2}}, \\
K_{A d S_{2}}^{\mathrm{f}}(t)=-\frac{1}{\pi} \int_{0}^{\infty} d v v \operatorname{coth}(\pi v) e^{-t v^{2}} .
\end{array}
$$

The gauge vector field contribution in (2.20) can be written as the difference of the contribution $K^{\mathrm{v}}$ of the operator $-G_{\alpha \beta} \nabla^{2}+R_{\alpha \beta}$ and that of the 2 scalar ghost operators [21]

$K^{\mathrm{g}}=K^{\mathrm{v}}-2 K^{\mathrm{s}}, \quad K^{\mathrm{v}}=K_{A d S_{2}}^{\mathrm{v}} K_{S^{2}}^{\mathrm{s}}+K_{S^{2}}^{\mathrm{v}} K_{A d S_{2}}^{\mathrm{s}}$.

The vector heat kernel on $S^{2}$ is the same as that of two scalar ones with 0 -mode subtracted, while on $A d S_{2}$ there is an additional vector mode that is not related to the scalar ones, i.e.,

$K_{S^{2}}^{\mathrm{v}}=2\left(K_{S^{2}}^{\mathrm{s}}-\frac{1}{4 \pi}\right), \quad K_{A d S_{2}}^{\mathrm{v}}=2 K_{A d S_{2}}^{\mathrm{s}}+\frac{1}{2 \pi}$.

As a result, $K^{\mathrm{g}}$ can be written as the contribution of two scalars plus an extra term:

$$
\begin{aligned}
K^{\mathrm{g}} & =2 K^{\mathrm{s}}+\tilde{K}, \quad K^{\mathrm{s}}=K_{A d S_{2}}^{\mathrm{s}}(t) K_{S^{2}}^{\mathrm{s}}(t), \\
\tilde{K} & =\frac{1}{2 \pi}\left[K_{S^{2}}^{\mathrm{s}}(t)-K_{A d S_{2}}^{\mathrm{s}}(t)\right] .
\end{aligned}
$$

The total traced heat kernel contribution of the fields of an Abelian $\mathcal{N}=4$ multiplet is then

$$
\begin{aligned}
K(t)= & 8 K_{A d S_{2}}^{\mathrm{s}}(t) K_{S^{2}}^{\mathrm{s}}(t)+4 K_{A d S_{2}}^{\mathrm{f}}(t) K_{S^{2}}^{\mathrm{f}}(t) \\
& +\frac{1}{2 \pi}\left[K_{S^{2}}^{\mathrm{s}}(t)-K_{A d S_{2}}^{\mathrm{s}}(t)\right] .
\end{aligned}
$$

From (A7)-(A9) we get then for the $\zeta$-function (A2) in the scalar operator case [using the expression for $V_{A d S_{2} \times S^{2}}$ in (2.22)]

$\zeta^{\mathrm{s}}(z)=-\sum_{\ell=0}^{\infty}(2 \ell+1) \int_{0}^{\infty} d v \frac{v \tanh (\pi v)}{\left[v^{2}+\left(\ell+\frac{1}{2}\right)^{2}\right]^{z}}$.

To evaluate this we may first $\operatorname{split} \tanh (\pi v)$ into two terms as $\tanh (\pi v)=1-2\left(e^{2 \pi v}+1\right)^{-1}$. Denoting the corresponding contributions as $\zeta_{1}^{\mathrm{s}}(z)$ and $\zeta_{2}^{\mathrm{s}}(z)$ we find

\footnotetext{
${ }^{11}$ Note that there is no scalar zero mode on the total $A d S_{2} \times S^{2}$ space despite the presence of $\ell=0$ zero mode on $S^{2}$.
} 
$1 / N$ CORRECTION IN THE D3-BRANE DESCRIPTION ...

$$
\begin{gathered}
\zeta_{1}^{\mathrm{s}}(0)=-\frac{7}{960}, \quad \zeta_{1}^{\mathrm{s}}(0)=-\frac{1}{960}\left[7-2 \ln 2+1680 \zeta_{R}^{\prime}(-3)\right] \\
\zeta_{2}^{\mathrm{s}}(z)=2 \int_{0}^{\infty} d v \frac{v}{e^{2 \pi v}+1} H(v, z), \\
H(v, z) \equiv \sum_{\ell=0}^{\infty} \frac{2 \ell+1}{\left[v^{2}+\left(\ell+\frac{1}{2}\right)^{2}\right]^{z}}, \\
\zeta_{2}^{\mathrm{s} \prime}(0)=-2 \int_{0}^{\infty} d v \frac{v}{e^{2 \pi v}+1} H^{\prime}(v, 0)
\end{gathered}
$$

where prime denotes derivative over $z$. Expanding the denominator of $H$ in power series in $v$ and doing the sum using the standard Riemann zeta function, i.e., $\sum_{\ell=0}^{\infty}(2 \ell+1)^{-p}=\left(1-2^{-p}\right) \zeta_{R}(p)$, etc., gives (see, e.g., [31-33])

$$
\begin{aligned}
H(v, z)= & \sum_{r=0}^{\infty}\left(-v^{2}\right)^{r} 2^{2 z+2 r} \frac{\Gamma(r+z)}{\Gamma(z) \Gamma(r+1)} \\
& \times \sum_{\ell=0}^{\infty}(2 \ell+1)^{-2 r-2 z+1} \\
= & 2 \sum_{r=0}^{\infty}\left(-v^{2}\right)^{r} \frac{\Gamma(r+z)}{\Gamma(z) \Gamma(r+1)}\left(2^{2 r+2 z-1}-1\right) \\
& \times \zeta_{R}(2 r+2 z-1) .
\end{aligned}
$$

As a result, ${ }^{12}$

$$
\begin{gathered}
H(v, 0)=\frac{1}{12}-v^{2}, \quad \text { (A21) } \\
H^{\prime}(v, 0)=-\frac{1}{6}(1+\ln 2)+2 \ln A \\
+\int_{0}^{v^{2}} d x\left[\psi\left(\frac{1}{2}+i \sqrt{x}\right)+\psi\left(\frac{1}{2}-i \sqrt{x}\right)\right] \\
=-\frac{1}{6}-\ln (2 \pi)-4 \ln A+2 \mathcal{H}(v), \quad(\mathrm{A} 22) \\
\mathcal{H}(v)=i v \ln \frac{\Gamma\left(\frac{1}{2}-i v\right)}{\Gamma\left(\frac{1}{2}+i v\right)}+\psi^{(-2)}\left(\frac{1}{2}-i v\right)+\psi^{(-2)}\left(\frac{1}{2}+i v\right) .
\end{gathered}
$$

Here $\psi^{(n)}(x)$ is the polygamma function, $\psi(x)=\psi^{(1)}(x)$, and $A$ is the Glaisher constant,

\footnotetext{
${ }^{12}$ We used the identity $\sum_{r=2}^{\infty}\left(-v^{2}\right)^{r}\left(2^{2 r-1}-1\right) r^{-1} \zeta_{R}(2 r-1)=$ $v^{2}\left(\gamma_{E}+2 \ln 2\right)+\frac{1}{2} \int_{0}^{v^{2}} d x\left[\psi\left(\frac{1}{2}+i \sqrt{x}\right)+\psi\left(\frac{1}{2}-i \sqrt{x}\right)\right]$.
}

$$
\begin{aligned}
\ln A & =\frac{1}{12}-\zeta_{R}^{\prime}(-1) \\
& =\frac{1}{12}\left[\gamma_{E}+\ln (2 \pi)\right]-\frac{1}{2 \pi^{2}} \zeta_{R}^{\prime}(2) \\
& =1.282 \ldots
\end{aligned}
$$

Thus $\zeta_{2}^{\mathrm{s}}(0)=-\frac{11}{2880}$ and

$$
\begin{aligned}
\zeta_{2}^{\mathrm{s}^{\prime}}(0) & =-\frac{1}{144}[1+6 \ln (2 \pi)+24 \ln A]+I_{1}, \\
I_{1} & \equiv 4 \int_{0}^{\infty} d v \frac{v}{e^{2 \pi v}+1} \mathcal{H}(v) .
\end{aligned}
$$

It is not clear how to compute the convergent integral $I_{1}$ in (A25) analytically, but it is straightforward to evaluate it numerically:

$$
I_{1}=0.117854 \ldots
$$

Combining (A17) and (A25) we finally get $\zeta^{\mathrm{s}}(0)=-\frac{1}{90}$ and

$$
\begin{aligned}
\zeta^{\mathrm{s} \prime}(0)= & -\frac{1}{2880}[41+114 \ln 2+120 \ln \pi \\
& \left.+480 \ln A+5040 \zeta_{R}^{\prime}(-3)\right]+I_{1},
\end{aligned}
$$

where $\zeta_{R}^{\prime}(-3)=0.005 \ldots$

In the Majorana fermion case (A10), (A11) we get

$K^{\mathrm{f}}(t)=-\frac{1}{4 \pi^{2}} \sum_{\ell=0}^{\infty}(2 \ell+2) e^{-t(\ell+1)^{2}} \int_{0}^{\infty} d v v \operatorname{coth}(\pi v) e^{-t v^{2}}$,

$\zeta^{\mathrm{f}}(z)=2 \sum_{\ell=0}^{\infty}(2 \ell+2) \int_{0}^{\infty} d v \frac{v \operatorname{coth}(\pi v)}{\left[v^{2}+(\ell+1)^{2}\right]^{z}}$.

The computation of $\zeta^{\mathrm{f}}(z)$ follows similar steps as above, i.e., splitting $\operatorname{coth}(\pi v)=1+2\left(e^{2 \pi v}-1\right)^{-1}$, etc. We find that $\zeta^{\mathrm{f}}(0)=-\frac{11}{180}$ and $^{13}$

$$
\begin{aligned}
\zeta^{\mathrm{f} \prime}(0) & =\frac{1}{180}\left[7-120 \ln A-720 \zeta_{R}^{\prime}(-3)\right]+2 I_{2}, \\
I_{2} & \equiv 4 \int_{0}^{\infty} d v \frac{v}{e^{2 \pi v}-1} \mathcal{G}(v), \\
\mathcal{G}(v) & =\frac{1}{2} \int_{0}^{v^{2}} d x[\psi(i \sqrt{x})+\psi(-i \sqrt{x})] \\
& =i v \ln \frac{\Gamma(-i v)}{\Gamma(i v)}+\psi^{(-2)}(-i v)+\psi^{(-2)}(i v) .
\end{aligned}
$$

\footnotetext{
${ }^{13}$ Here we used that $\sum_{r=2}^{\infty}\left(-v^{2}\right)^{r} r^{-1} \zeta_{R}(2 r-1)=$ $\gamma_{E} v^{2}+\frac{1}{2} \int_{0}^{v^{2}} d x[\psi(i \sqrt{x})+\psi(-i \sqrt{x})]$.
} 
The numerical value of $I_{2}$ is

$$
I_{2}=0.237101 \ldots
$$

To determine the vector contribution (A14) to $\zeta^{\prime}(0)$ we need to add to the two scalar contributions (A27) an extra term corresponding to

$\tilde{K}=-\frac{1}{2 \pi} K_{A d S_{2}}^{\mathrm{s}}(t)+\frac{1}{2 \pi} K_{S^{2}}^{\mathrm{s}}(t) \equiv \tilde{K}_{1}(t)+\tilde{K}_{2}(t)$,

where $K_{S^{2}}^{\mathrm{s}}$ and $K_{A d S_{2}}^{\mathrm{s}}$ were given in (A8) and (A9). The $\zeta$-function corresponding to the $A d S_{2}$ part $\tilde{K}_{1}(t)$ is

$$
\begin{gathered}
\tilde{\zeta}_{1}(z)=2 \int d v \frac{v \tanh (\pi v)}{\left(v^{2}+\frac{1}{4}\right)^{z}}, \quad \tilde{\zeta}_{1}(0)=-\frac{1}{3}, \quad \mathrm{~A} \\
\tilde{\zeta}_{1}^{\prime}(0)=\frac{1}{6}(1+\ln 2)-2 \ln A+2 \int_{0}^{1 / 4} d x \psi\left(\sqrt{x}+\frac{1}{2}\right) \\
=\frac{1}{6}-\ln (2 \pi)+4 \ln A .
\end{gathered}
$$

The $S^{2}$ part contains the IR singular zero-mode $(\ell=0)$ contribution. It may be regularized by adding a small mass parameter $\mathrm{m}^{2}$,

$$
\begin{aligned}
& \tilde{K}_{2}(t)=\frac{1}{8 \pi^{2}} \sum_{\ell=0}^{\infty}(2 \ell+1) e^{-s\left[\ell(\ell+1)+\mathrm{m}^{2}\right]}, \\
& \tilde{\zeta}_{2}(z)=-\sum_{\ell=0}^{\infty} \frac{2 \ell+1}{\left[\left(\ell+\frac{1}{2}\right)^{2}+\left(\mathrm{m}^{2}-\frac{1}{4}\right)\right]^{z}} .
\end{aligned}
$$

As a result,

$$
\begin{aligned}
\tilde{\zeta}_{2}^{\prime}(0)= & \frac{1}{6}(1+\ln 2)-2 \ln A \\
& -\int_{0}^{-1 / 4+\mathrm{m}^{2}} d x\left[\psi\left(\frac{1}{2}+i \sqrt{x}\right)+\psi\left(\frac{1}{2}-i \sqrt{x}\right)\right] .
\end{aligned}
$$

Taking the limit $\mathrm{m} \rightarrow 0$ and omitting the singular $\ln \mathrm{m}$ term we get

$\tilde{\zeta}_{2}^{\prime}(0)=\frac{1}{6}+4 \ln A+\ln \mathrm{m}^{2} \rightarrow \frac{1}{6}+4 \ln A=\frac{1}{2}-4 \zeta_{R}^{\prime}(-1)$.

This is the same expression as found in [34]. Note that including formally (the regularized) zero-mode contribution in the $\zeta$-function gives $\tilde{\zeta}_{2}(0)=-\frac{1}{3}$ which matches the value of the corresponding Seeley coefficient. Summing up the expressions for $\tilde{\zeta}_{1}$ and $\tilde{\zeta}_{2}$ in (A35) and (A38) we get $\tilde{\zeta}(0)=-\frac{2}{3}$ and

$$
\tilde{\zeta}^{\prime}(0)=\frac{1}{3}-\ln (2 \pi)+8 \ln A \text {. }
$$

We can now combine the above results to find the total values of $\zeta(0)$ and $\zeta^{\prime}(0)$ for the $\mathcal{N}=4$ supersymmetric Abelian gauge theory in $A d S_{2} \times S^{2}$ background. Explicitly, for the sum of the gauge field $\zeta^{\mathrm{g}}=\zeta^{\mathrm{v}}-2 \zeta^{\mathrm{s}}=2 \zeta^{\mathrm{s}}+\tilde{\zeta}$ [cf. (A14)], 6 real scalar $\zeta^{\mathrm{s}}$ and 4 Majorana fermion $\zeta^{\mathrm{f}}$ contributions, i.e., $\zeta=\zeta^{\mathrm{g}}+6 \zeta^{\mathrm{s}}+4 \zeta^{\mathrm{f}}=8 \zeta^{\mathrm{s}}+4 \zeta^{\mathrm{f}}+\tilde{\zeta}$, we get

$$
\begin{gathered}
\zeta(0)=8 \zeta^{\mathrm{s}}(0)+4 \zeta^{\mathrm{f}}(0)+\tilde{\zeta}(0) \\
=-8 \times \frac{1}{90}-4 \times \frac{11}{180}-\frac{2}{3} \\
=-1, \\
\zeta^{\prime}(0)=8 \zeta^{\prime \mathrm{s}}(0)+4 \zeta^{\prime \mathrm{f}}(0)+\tilde{\zeta}^{\prime}(0) \\
=-\frac{1}{120}(-45+158 \ln 2+160 \ln \pi)+4 \ln A \\
-30 \zeta_{R}^{\prime}(-3)+8\left(I_{1}+I_{2}\right) .
\end{gathered}
$$

The integrals $I_{1}$ and $I_{2}$ were given in (A25), (A26) and (A30), (A32).

We conclude that the value of $\zeta(0)$ is the same as of $B_{4}$ in (2.22) and the constant parameter-independent part $C_{1}$ of the 1-loop effective action (1.9) is proportional to (A41) [cf. (A3)]. Its numerical value is

$$
C_{1}=-\frac{1}{2} \zeta^{\prime}(0)=-0.809684 \ldots
$$

\section{APPENDIX B: THE $A d S_{5} \times S^{5}$ STRING ONE-LOOP CORRECTION TO CIRCULAR WILSON LOOP REVISITED}

For completeness, let us review the expression for the $A d S_{5} \times S^{5}$ string one loop correction to the ( $k$-wound) circular Wilson loop $[10,11]$ found in the limit when one first takes $N=\infty$ and then expands in large string tension $\sqrt{\lambda}$ for fixed $k$. Here one represents the planar expectation value $\langle W\rangle=e^{-\bar{\Gamma}}, \bar{\Gamma}=\bar{\Gamma}_{0}+\bar{\Gamma}_{1}+\bar{\Gamma}_{2}+\cdots$, by the string path integral with a disclike world sheet ending on a circle at the boundary of $A d S_{5} \times S^{5}$ space. The classical world-sheet metric is that of the ( $k$-wrapped version of) Euclidean $A d S_{2}$ with $S^{1}$ boundary and so the classical action [proportional to the renormalized volume in (2.9)] is $\bar{\Gamma}_{0}=-k \sqrt{\lambda}$, while $\bar{\Gamma}_{1}=\Gamma_{1}(k), \bar{\Gamma}_{2}=\frac{1}{\sqrt{\lambda}} \tilde{\Gamma}_{2}(k)$, etc.

The general form of the conformal-gauge string one loop correction was given in [23] as

$$
\bar{\Gamma}_{1}=\frac{1}{2} \ln \frac{\left[\operatorname{det}\left(-\nabla^{2}+2\right)\right]^{3}\left[\operatorname{det}\left(-\nabla^{2}\right)\right]^{5}}{\left[\operatorname{det}\left(-\nabla^{2}+\frac{1}{4} R+1\right)\right]^{8}} .
$$


In the straight line WL case the classical action and loop corrections are proportional to the volume of $\widetilde{A d S_{2}}$ with boundary $R$ which has zero renormalized value (2.9) and thus they should be assumed to vanish [23,24], in agreement with $\langle W\rangle=1$ on the gauge theory side.

In the circular case the computation of the corresponding determinants was carried out using different methods in $[23,24,35]$, with the finite part of the resulting expression for $\bar{\Gamma}_{1}(k)$ being [24]

$$
\begin{aligned}
& \bar{\Gamma}_{1 \text { fin }}(k)=\bar{\Gamma}_{1 \text { fin }}(1)+\left(2 k+\frac{1}{2}\right) \ln k-\ln k !, \\
& \bar{\Gamma}_{1 \text { fin }}(1)=\frac{1}{2} \ln (2 \pi) .
\end{aligned}
$$

At the same time, the gauge theory expression (1.4) taken at $N \rightarrow \infty$ and then expanded in large $\lambda$ for fixed $k$ gives the familiar modified Bessel function expression $[1,12,13]$

$$
\begin{gathered}
\langle W\rangle=2(k \sqrt{\lambda})^{-1} I_{1}(k \sqrt{\lambda})=e^{k \sqrt{\lambda}-\Gamma_{1}+\cdots}, \text { where } \\
\Gamma_{1}=\hat{\Gamma}_{1}+\frac{3}{2} \ln k+\frac{3}{2} \ln \sqrt{\lambda}, \quad \hat{\Gamma}_{1}=\frac{1}{2} \ln \frac{\pi}{2} .
\end{gathered}
$$

Here the second $\frac{3}{2} \ln k$ term is of course the same as the one present in (1.8). The third term may be attributed to the presence of the string tension normalization factor for the three (Mobius-symmetry) ghost zero modes on the disc [13], which was not included in (B1), (B2). Even ignoring this term, $\bar{\Gamma}_{1 \text { fin }}(1)$ in (B2) still differs from $\hat{\Gamma}_{1}$ in (B3) by an extra ln 2 term. This difference may be coming from a numerical factor in normalization of the disc zero modes or from the ratio of the ghost and the two longitudinal mode determinants [assumed to be equal to one in (B1)] once they are computed with proper boundary conditions (cf. [35]).

A resolution of this problem may lead to a change in the $k$-dependence of $\Gamma_{1 \text { fin }}$ in (B2) making it match the second term in the gauge-theory result (B3). Here we will not attempt to resolve this issue and will only review and complete the original computation in [23] of $\Gamma_{1 \text { fin }}$ in (B2) for $k=1$ based on expressing the determinants in (B1) in terms of the known $[19,20]$ heat kernels of the scalar and spinor Laplacians on $A d S_{2}$. This provides an alternative to the derivation of $\Gamma_{1 \text { fin }}$ in [24].

The discussion below repeats the computation in Appendix B.1 in [23]. There it was assumed to apply to the straight string case and it was not appreciated that in the case of $\widetilde{A d S}_{2}$ with the boundary $R$ the result, proportional to the volume $V_{\widetilde{A d S} S_{2}} \sim \frac{1}{\varepsilon}$ contains the only IR divergent piece that may be discarded [24]. The expressions in Appendix B.1 in [23] are, in fact, literally valid in the circular string case where the classical world sheet metric is that of $A d S_{2}$ with $S^{1}$ boundary which has finite renormalized volume (2.9). ${ }^{14}$

$\bar{\Gamma}_{1}$ in (B1) contains the contributions of 3 scalars with mass-squared $m^{2}=2,5$ scalars with $m^{2}=0$ and 8 Majorana fermions with $m^{2}=1$, propagating in $A d S_{2}$. We will set the $A d S_{2}$ radius to 1 since $\bar{\Gamma}_{1}$ does not depend on it (assuming UV divergences eventually cancel [23]). As in (A1) here

$\frac{1}{2} \ln \operatorname{det} \Delta=-\frac{1}{2} V_{A d S_{2}} \int_{\epsilon}^{\infty} \frac{d t}{t} K(t), \quad V_{A d S_{2}}=-2 \pi$.

The trace of heat kernel for massive scalars and fermions may be written as

$$
K(t)=\frac{1}{2 \pi} \int_{0}^{\infty} d v \mu(v) e^{-t\left(v^{2}+M\right)},
$$

$\mu(v)=v \tanh (\pi v), \quad M=\frac{1}{4}+m^{2} \quad$ for scalar,

$\mu(v)=-v \operatorname{coth}(\pi v), \quad M=m^{2} \quad$ for Majorana fermion.

The corresponding $\zeta$-function is [cf. (A2)]

$$
\begin{aligned}
\zeta(z) & =-\frac{1}{\Gamma(z)} \int_{0}^{\infty} d v \mu(v) \int_{0}^{\infty} d t t^{z-1} e^{-t\left(v^{2}+M\right)} \\
& =-\int_{0}^{\infty} d v \frac{\mu(v)}{\left(v^{2}+M\right)^{z}} .
\end{aligned}
$$

Starting with the scalar case we may split the spectral density as $\tanh (\pi v)=1-2\left(e^{2 \pi v}+1\right)^{-1}$. The contribution from the first term to $\zeta^{\prime}(0, M)$ is then $-\frac{1}{2} M(\ln M-1)$. The second term gives an exponentially convergent integral for large $v$ so we can set $z=0$ inside the integral. Then the scalar $\zeta$-function is

$$
\begin{aligned}
\zeta^{\prime}(0, M) & =-\frac{1}{2} M(\ln M-1)-J_{1}(M), \\
J_{1}(M) & \equiv 2 \int_{0}^{\infty} d v \frac{v \ln \left(v^{2}+M\right)}{e^{2 \pi v}+1} .
\end{aligned}
$$

We may write $J_{1}(M)=\int_{0}^{M} d x \frac{\partial J_{1}(x)}{\partial x}+J_{1}(0)$ where [20]

\footnotetext{
${ }^{14}$ The expressions for heat kernels and $\zeta$-functions there did not contain the volume factor $V_{A d S_{2}}=-2 \pi$ which we will include below. We will also perform the final step of summation of the bosonic and fermionic contributions that was not done explicitly in Appendix B.1 in [23].
} 


$$
\begin{gathered}
\frac{\partial J_{1}(M)}{\partial M}=2 \int_{0}^{\infty} d v \frac{v}{\left(e^{2 \pi v}+1\right)\left(v^{2}+M\right)} \\
=-\frac{1}{2} \ln M+\psi\left(\sqrt{M}+\frac{1}{2}\right), \\
J_{1}(0)=2 \int_{0}^{\infty} d v \frac{v \ln v^{2}}{e^{2 \pi v}+1}=\frac{1}{12}(1+\ln 2)-\ln A .
\end{gathered}
$$

Thus

$\zeta^{\mathrm{s}}(0, M)=-\frac{1}{12}(1+\ln 2)+\ln A-\int_{0}^{M} d x \psi\left(\sqrt{x}+\frac{1}{2}\right)$,

where $A$ is the Glaisher constant (A24). The total bosonic contribution of 3 scalars [see (B6)] with $M=\frac{1}{4}+2=\frac{9}{4}$ and 5 scalars with $M=\frac{1}{4}$ is found (after doing the integrals) to be

$$
\begin{aligned}
\zeta_{B}^{\prime}(0) & =3 \zeta^{\mathrm{s} \prime}\left(0, \frac{9}{4}\right)+5 \zeta^{\mathrm{s} \prime}\left(0, \frac{1}{4}\right) \\
& =-\frac{20}{3}+7 \ln (2 \pi)-16 \ln A
\end{aligned}
$$

Performing a similar computation in the fermionic case (B7) [with $\operatorname{coth}(\pi \lambda)=1+2\left(e^{2 \pi \lambda}-1\right)^{-1}$, etc.] we get

$$
\begin{aligned}
\zeta^{\mathrm{f} \prime}(0, M) & =\frac{1}{2} M(\ln M-1)-J_{2}(M), \\
J_{2}(M) & =2 \int_{0}^{\infty} d v \frac{v \ln \left(v^{2}+M\right)}{e^{2 \pi v}-1}, \\
\frac{\partial J_{2}(M)}{\partial M} & =2 \int_{0}^{\infty} d v \frac{v}{\left(e^{2 \pi v}-1\right)\left(v^{2}+M\right)} \\
& =\frac{1}{2} \ln M-\frac{1}{2}(\sqrt{M})^{-1}-\psi(\sqrt{M}), \\
\zeta^{\mathrm{f}}(0, M)= & -\frac{1}{6}+2 \ln A+\sqrt{M}+\int_{0}^{M} d x \psi(\sqrt{x}) .
\end{aligned}
$$

The contribution of 8 Majorana fermions with $M=m^{2}=1$ is then

$$
\zeta_{F}^{\prime}(0)=8 \zeta^{\mathrm{f} \prime}(0,1)=\frac{20}{3}-8 \ln (2 \pi)+16 \ln A .
$$

Thus the total 1-loop correction coming from (B1) is

$$
\Gamma_{1}=-\frac{1}{2} \zeta_{B}^{\prime}(0)-\frac{1}{2} \zeta_{F}^{\prime}(0)=\frac{1}{2} \ln (2 \pi),
$$

which is indeed the same as $\Gamma_{1 \text { fin }}$ in (B2).

It should be possible to generalize the above computation to the case of $k$-wrapped circular Wilson loop to check the result (B2) of [24]. The corresponding solution in $A d S_{3}$ with the metric $d s^{2}=z^{-2}\left(d r^{2}+r^{2} d \phi^{2}+d z^{2}\right)$ is described by $\phi=k \tau, z=\tanh k \sigma, r=\cosh ^{2} k \sigma, z=\sqrt{1-r^{2}}$, where $\tau \in(0,2 \pi), \sigma \in(0, \infty)$. The induced metric is $d s^{2}=k^{2}(\sinh \kappa \sigma)^{-2}\left(d \tau^{2}+d \sigma^{2}\right)=d \xi^{2}+k^{2} \sinh ^{2} \xi d \tau^{2}$, where $e^{\xi}=\tanh \frac{k \sigma}{2}$. For $k=1$ this is the standard regular $A d S_{2}$ metric but for general $k$ it has a conical singularity at $\xi=0$ with negative deficit $\delta=2 \pi(1-k), k=2,3,4, \ldots$. While we are interested in the case of integer $k$ let us formally consider $k$ as an arbitrary real number. Then setting it to be $1 / n$ with an integer $n$ we get an orbifold $A d S_{2} / Z_{n}$ (with a conical singularity of positive deficit) and the corresponding heat kernel can be found as a sum over images $[22,30,36] .{ }^{15}$ In the case of a cone of a 2-plane $d s^{2}=$ $d \xi^{2}+k^{2} \xi^{2} d \tau^{2}$ with generic $k$ the heat kernel can be found using "reperiodization" trick [37] and the same idea applies to the cone of $A d S_{2}$ with explicit expression for the scalar case given in [36]. The analogous expression for the spinor heat kernel for generic $k$ can be found using the results of $[20,38]$ and following the examples of the cones of 2-plane and 2-sphere in [39]. We leave a detailed computation for an integer $k$ for the future.

\footnotetext{
${ }^{15}$ Explicitly, it is given by the following modification of (A5): $\quad K_{A d S_{2}}\left(x, x^{\prime} ; t\right)=\frac{1}{2 \pi} \sum_{r=0}^{n-1} \int_{0}^{\infty} d v v \tanh (\pi v) P_{-\frac{1}{2}+i v} \times$ $\left(\cosh d\left(\xi, \tau ; \xi^{\prime}, \tau^{\prime}+\frac{2 \pi r}{n}\right)\right) e^{-t\left(v^{2}+\frac{1}{4}\right)}$. Similar construction applies in the spinor case [22].
}

[1] N. Drukker and B. Fiol, J. High Energy Phys. 02 (2005) 010.

[2] J. Gomis and F. Passerini, J. High Energy Phys. 08 (2006) 074; J. Gomis and F. Passerini, J. High Energy Phys.01 (2007) 097.

[3] S. A. Hartnoll and S. P. Kumar, J. High Energy Phys. 08 (2006) 026.

[4] K. Okuyama and G. W. Semenoff, J. High Energy Phys. 06 (2006) 057.
[5] S. Yamaguchi, J. High Energy Phys. 06 (2007) 073.

[6] N. Drukker, S. Giombi, R. Ricci, and D. Trancanelli, J. High Energy Phys. 04 (2007) 008.

[7] H. Lin, O. Lunin, and J. M. Maldacena, J. High Energy Phys. 10 (2004) 025.

[8] S. Yamaguchi, Int. J. Mod. Phys. A 22, 1353 (2007).

[9] O. Lunin, J. High Energy Phys. 06 (2006) 026. 
$1 / N$ CORRECTION IN THE D3-BRANE DESCRIPTION ...

[10] D. E. Berenstein, R. Corrado, W. Fischler, and J. M. Maldacena, Phys. Rev. D 59, 105023 (1999).

[11] N. Drukker, D. J. Gross, and H. Ooguri, Phys. Rev. D 60, 125006 (1999).

[12] J. K. Erickson, G. W. Semenoff, and K. Zarembo, Nucl. Phys. B582, 155 (2000).

[13] N. Drukker and D. J. Gross, J. Math. Phys. (N.Y.) 42 (2001) 2896.

[14] V. Pestun, Commun. Math. Phys. 313, 71 (2012).

[15] B. Fiol and G. Torrents, J. High Energy Phys. 01 (2014) 020.

[16] S. Kawamoto, T. Kuroki, and A. Miwa, Phys. Rev. D 79, 126010 (2009).

[17] A. Faraggi and L. A. Pando Zayas, J. High Energy Phys. 05 (2011) 018.

[18] A. Faraggi, W. Mueck, and L. A. Pando Zayas, Phys. Rev. D 85, 106015 (2012).

[19] R. Camporesi, Phys. Rep. 196 (1990) 1.

[20] R. Camporesi, Phys. Rev. D 43, 3958 (1991); Commun. Math. Phys. 148, 283 (1992); R. Camporesi and A. Higuchi, Phys. Rev. D 47, 3339 (1993); J. Math. Phys. (N.Y.) 35, 4217 (1994).

[21] S. Banerjee, R. K. Gupta, and A. Sen, J. High Energy Phys. 03 (2011) 147; S. Banerjee, R. K. Gupta, I. Mandal, and A. Sen, J. High Energy Phys. 11 (2011) 143.

[22] R. K. Gupta, S. Lal, and S. Thakur, J. High Energy Phys. 03 (2014) 043; arXiv:1402.2441.

[23] N. Drukker, D. J. Gross, and A. A. Tseytlin, J. High Energy Phys. 04 (2000) 021.
PHYSICAL REVIEW D 89, 126008 (2014)

[24] M. Kruczenski and A. Tirziu, J. High Energy Phys. 05 (2008) 064.

[25] G. W. Semenoff and K. Zarembo, Nucl. Phys. B, Proc. Suppl. 108, 106 (2002).

[26] N. Seiberg and E. Witten, J. High Energy Phys. 09 (1999) 032.

[27] M. J. Duff, Nucl. Phys. B125, 334 (1977).

[28] D. V. Vassilevich, Phys. Rep. 388, 279 (2003).

[29] I. Chavel, Heat Kernels and Spectral Theory (Cambridge University Press, Cambridge, England, 1984).

[30] T. H. Jones and D. Kucerovsky, arXiv:1007.5467; T. H. Jones, Ph.D. thesis, University of New Brunswick, 2008.

[31] S. M. Christensen and M. J. Duff, Nucl. Phys. B170, 480 (1980).

[32] E. S. Fradkin and A. A. Tseytlin, Nucl. Phys. B234, 472 (1984).

[33] N. Sakai and Y. Tanii, Nucl. Phys. B255, 401 (1985).

[34] W. I. Weisberger, Nucl. Phys. B284, 171 (1987).

[35] C. Kristjansen and Y. Makeenko, J. High Energy Phys. 09 (2012) 053.

[36] R. B. Mann and S. N. Solodukhin, Nucl. Phys. B523, 293 (1998).

[37] J. S. Dowker, J. Phys. A 10, 115 (1977); D. V. Fursaev, Classical Quantum Gravity 11, 1431 (1994); J. S. Dowker, Phys. Rev. D 50, 6369 (1994).

[38] R. Camporesi and A. Higuchi, J. Geom. Phys. 20, 1 (1996).

[39] D. V. Fursaev and G. Miele, Nucl. Phys. B484, 697 (1997). 